was significant intra-individual variation of $\mathrm{FC}$ within $\mathrm{CD}$ patients, this could impact on the interpretation of single values in isolation. A prospective study to evaluate the degree of variability of FC in three consecutive days stool samples in $\mathrm{CD}$ patients in clinical remission was performed.

Methods 143 patients with CD in remission (CDAI $<150$, no escalation of medical therapy within 3 months) who were attending for routine follow-up were identified and enrolled. Patients were excluded if they were taking NSAIDs or developed any change in symptoms over the 3-day collection period. After informed consent, patients were asked to provide stool samples from three consecutive days for analysis. FC was analysed using the Buhlman FC ELISA as per the manufacturer's instructions.

Results Of the 143 patients recruited 34 did not return any samples, 6 returned less than the required number of stool samples, 2 withdrew consent and 2 developed clinical evidence of a flare of disease during the collection period and were excluded from analysis. Therefore 98 complete sets of three stool samples were obtained and analysed. The intraclass correlation coefficient of the $3 \mathrm{FC}$ values, log-transformed, was 0.842 with corresponding $95 \%$ CI 0.788 to 0.886 showing that consistency between the three log-transformed FC measurements is high. The reliability of a "normal" FC result of $<50$ and of $<100$ was assessed using the $\kappa$ statistic for agreement between the three measurements. For a FC result of $<50 \kappa$ was $0.648(95 \%$ CI 0.508 to 0.770$)$ and for a FC result of $<100, \kappa$ was 0.603 (95\% CI 0.483 to 0.712 ) demonstrating that there is moderately good and similar levels of agreement across the 3 samples for both measures. The reliability of the cut-off of 50 is slightly better than the cut-off of 100 .

Conclusion The consistency of three faecal calprotectin samples over three consecutive days is high and the $\mathrm{CI}$ is narrow suggesting that in a larger population consistency would also be high. This indicates that there is little intra-individual variability of the test and a one off sample can indeed provide reliable information for use in clinical practice. Higher intrapatient variability of high $\mathrm{FC}$ values suggests that the log-transformed values are more reliable.

Competing interests None declared.

\section{PTU-107 WEIGHT ADJUSTED ANTI-TNF THERAPY FAVOURS OBESE PATIENTS WITH CROHN'S DISEASE}

doi:10.1136/gutjnl-2012-302514c.107

${ }^{1} \mathrm{M}$ Bhalme, ${ }^{*} \mathrm{~A}$ Sharma, ${ }^{3} \mathrm{R}$ Keld, ${ }^{4} \mathrm{~A}$ Makin, ${ }^{4} \mathrm{R}$ Willert, ${ }^{4} \mathrm{~S}$ Campbell. ${ }^{1}$ North Manchester General Hospital, Manchester, Manchester, UK; ${ }^{2}$ Royal Preston Hospital, Preston, UK; ${ }^{3}$ Royal Albert Edward Infirmary, Wigan, UK; ${ }^{4}$ Manchester Royal Infirmary, Manchester, UK

Introduction Adalimumab (Humira, Abbott) is a novel subcutaneous anti-TNF agent, effective in inducing and maintaining remission in Crohn's disease (CD). Unlike Infliximab (IFx), Adalimumab (Ad) dosing is not weight adjusted, and dose frequency is based on clinical response. Pharmacokinetic analyses in rheumatoid arthritis patients (pts) has shown weight to have minimal effect on Ad clearance. Our aim was to determine whether weight is important in predicting efficacy of weight adjusted and non-weight adjusted anti-TNF treatment in CD.

Methods A hospital database of CD patients receiving anti-TNF therapy was analysed retrospectively. Demographics, previous IFx exposure, disease anatomy, concomitant immunosuppressive therapy, smoking status and duration of anti-TNF treatment were recorded. A body mass index (BMI) cut off of $<30$ or $\geq 30$ was used to define obesity and time to dose escalation (increased frequency) and survival curve analysis were compared using Kaplan-Meier (KM) estimation. $\mathrm{p}<0.05$ was taken as significant. Analysis were made using Graphpad prism software.
Results Adalimumab patients: 54 (36 female; age range 17-72 years, median 38.5; BMI range 17.2-47.7, median 24.8) patients details were available. 46 patients had $\mathrm{BMI}<30$ and 8 had $\mathrm{BMI} \geq 30$. $\mathrm{CD}$ distribution and activity were heterogeneous. There was no significant difference $(\mathrm{p}=\mathrm{NS})$ in the duration of Ad treatment in $\mathrm{BMI}<30$ (range 1-39 months, median 10.5) vs $\mathrm{BMI} \geq 30$ (range 6-27 months, median 13.5). KM estimation revealed a significantly lower time to Ad dose escalation in the $\mathrm{BMI} \geq 30\left(\chi^{2} 6.117, \mathrm{p}=0.0134\right)$. There was no difference in time to dose escalation according to disease anatomy or smoking status. Infliximab patients: 76 (42 female; age range 11-70 years, median 37.5; BMI range 14.9-43.7, median 23.7) patients details were available. 62 patients had $\mathrm{BMI}<30$ and 14 had $\mathrm{BMI} \geq 30$. $\mathrm{CD}$ distribution and activity were heterogeneous. KM estimation revealed the survival curves for the IFx patients were close and statistically non-significant $\left(\chi^{2} 1.933\right.$, $\mathrm{p}=\mathrm{NS}$ ), in $\mathrm{BMI}<30$ and $\mathrm{BMI} \geq 30$.

Conclusion Patients BMI appears important in predicting Adalimumab efficacy in $\mathrm{CD}$ with respect to loss of response (LOR), irrespective of induction dosing. Weight adjusted anti-TNF treatment appears to overcome this apparent reduction of efficacy in obese patients, as demonstrated by the data. A trend for separation between the two groups may be explained by the additional effects of the pro-inflammatory adipokines in obese patients with CD. A prospective study on the effect of weight on drug response is warranted.

Competing interests None declared.

\section{PTU-108 INDUCTION OF REMISSION WITH ADALIMUMAB IN PATIENTS WITH MODERATE CROHN'S DISEASE: SUBANALYSIS OF CLASSIC I}

doi:10.1136/gutjnl-2012-302514c.108

${ }^{1} \mathrm{~W}$ J Sandborn, ${ }^{2} \mathrm{~J}$-F Colombel, ${ }^{3} \mathrm{M}$ M Castillo, ${ }^{*} \mathrm{O}$ Zhou, ${ }^{3} \mathrm{R}$ B Thakkar. ${ }^{1}$ Gastroenterology, University of California San Diego, San Diego, California, USA; ${ }^{2}$ Hepatogastroenterology, Centre Hospitalier Universitaire de Lille, Lille, France; ${ }^{3}$ Abbott, Abbott Park, USA

Introduction CLASSIC I, ${ }^{1}$ a randomised, double-blind, placebocontrolled, dose-ranging trial, demonstrated that adalimumab (ADA) $160 \mathrm{mg}$ at week 0 , followed by $80 \mathrm{mg}$ at week 2 is the optimal induction regimen in anti-TNF-naïve patients with moderate to severe Crohn's disease (CD). It also showed that patients with baseline $\mathrm{C}$ reactive protein (CRP) concentration $\geq 10 \mathrm{mg} / \mathrm{l}$ achieved higher rates of clinical remission (CDAI score $<150)$ at week 4.

Methods This post hoc analysis evaluated efficacy in patients with moderate CD (baseline CDAI $\leq 300$ ) and whether elevated CRP $(\geq 10 \mathrm{mg} / \mathrm{l})$ in this subgroup would similarly improve efficacy, compared with results for patients with severe CD. Clinical remission at week 4 was assessed for patients who received induction with ADA (160 mg/80 mg or $80 \mathrm{mg} / 40 \mathrm{mg}$ ) or placebo in CLASSIC I. Patients with the following characteristics were included in the analysis: baseline $\mathrm{CRP} \geq 10 \mathrm{mg} / \mathrm{l}$; moderate $\mathrm{CD}$ (baseline CDAI $\leq 300$ ); moderate $\mathrm{CD}$ with baseline $\mathrm{CRP} \geq 10 \mathrm{mg} / \mathrm{l}$; severe $\mathrm{CD}$ (baseline CDAI >300); and severe CD with baseline CRP $\geq 10 \mathrm{mg} / \mathrm{l}$. Results In CLASSIC I, ADA $160 \mathrm{mg} / 80 \mathrm{mg}$ was effective at inducing remission in all subgroups studied, including the subgroup of patients with moderate $\mathrm{CD}$; in this subgroup, high baseline CRP was associated with substantially higher remission rates. This analysis suggests that patients with moderate disease can be treated effectively with adalimumab, especially when there is evidence of inflammation. Prospective studies are warranted to confirm these findings.

Conclusion In CLASSIC I, ADA $160 \mathrm{mg} / 80 \mathrm{mg}$ was effective at inducing remission in all subgroups studied, including the subgroup 